\title{
Effect of fibre surface modification on properties of kenaf/poly(vinyl alcohol) composite film
}

\begin{abstract}
Kenaf/poly(vinyl alcohol) (PVA) composite films having 5, 10, 15\% wt\% of fibre loadings were pre-pared via aqueous mixing. The mixture was casted as composite film and it was characterized. Tensile properties, Fourier transform infra-red (FTIR) and morphology analyses were reported. The effect of fibre surface modification on the properties of composite film was investigated in the study. The result of citric acid modification was much better than the traditional mercerization of fibres via sodium hydroxide solution. The biodegradability of the composite films was evaluated by soil burial test. The soil burial test revealed that the modified Kenaf/PVA composite film was biodegradable in nature environment. It achieved the highest $\sim 8.91 \%$ reduction after 15 days buried under soil and exposed to environmental condition.
\end{abstract}

Keyword: Biodegradable; Composite; Kenaf; Poly(vinyl) alcohol; Tensile properties 